\title{
Manual vs. computer-assisted sperm analysis: can CASA replace manual assessment of human semen in clinical practice?
}

\author{
Joanna Talarczyk-Desole ${ }^{1}$, Anna Berger ${ }^{1}$, Grażyna Taszarek-Hauke ${ }^{1}$, \\ Jan Hauke ${ }^{2}$, Leszek Pawelczyk ${ }^{1}$, Piotr Jedrzejczak ${ }^{1}$ \\ ${ }^{1}$ Division of Infertility and Reproductive Endocrinology, Poznan University of Medical Sciences, Poland \\ ${ }^{2}$ Institute of Socio-Economic Geography and Spatial Management, Adam Mickiewicz University Poznan, Poland
}

\begin{abstract}
Objectives: The aim of the study was to check the quality of computer-assisted sperm analysis (CASA) system in comparison to the reference manual method as well as standardization of the computer-assisted semen assessment.

Material and methods: The study was conducted between January and June 2015 at the Andrology Laboratory of the Division of Infertility and Reproductive Endocrinology, Poznań University of Medical Sciences, Poland. The study group consisted of 230 men who gave sperm samples for the first time in our center as part of an infertility investigation. The samples underwent manual and computer-assisted assessment of concentration, motility and morphology. A total of 184 samples were examined twice: manually, according to the 2010 WHO recommendations, and with CASA, using the program settings provided by the manufacturer. Additionally, 46 samples underwent two manual analyses and two computer-assisted analyses. The $p$-value of $p<0.05$ was considered as statistically significant.

Results: Statistically significant differences were found between all of the investigated sperm parameters, except for non-progressive motility, measured with CASA and manually. In the group of patients where all analyses with each method were performed twice on the same sample we found no significant differences between both assessments of the same probe, neither in the samples analyzed manually nor with CASA, although standard deviation was higher in the CASA group.

Conclusions: Our results suggest that computer-assisted sperm analysis requires further improvement for a wider application in clinical practice.
\end{abstract}

Key words: computer-assisted sperm analysis, CASA, sperm parameters, infertility

Ginekologia Polska 2017; 88, 2: 56-60

\section{INTRODUCTION}

Seminological assessment constitutes the basic examination in the early diagnosis of male infertility [1]. Since 1980, the World Health Organization (WHO) has been publishing recommendations for proper sperm analysis. These recommendations have continued to be evaluated until 2010, when the 5 th edition of the WHO Laboratory Manual for the Examination and Processing of Human Semen was released [2], describing in detail all aspects of laboratory analysis of sperm parameters and preparation of the semen for the assisted reproduction. The WHO recommends manual sperm analysis as a standard and reference method of seminological examination.
Strict adherence to the guidelines in the WHO Manual results in obtaining the most standardized and objective values. In order to meet the requirements, intensive specialist training and quality control programs are needed. Unfortunately, adequate seminological assessment remains problematic in many laboratories. Methodology lapses lead to mistakes. For example in order to maximize repeatability of the results, manual analysis of sperm concentration should be performed with the Neubauer hemocytometer. Furthermore, accurate pipetting and dilution are needed. Nevertheless, some laboratories, in order to save time and expenses, use methods other than standard, i.e. different 
chambers or inadequate dilutions, which often leads to false results [3,4]. Also sperm motility assessment presents a challenge, not only in terms of the subjective analysis of gamete velocity, but also standardized temperature of the analysis or time elapsed between sample collection and the result [5]. Taking into account the inaccuracies in the examination processes, as well as lack of internal and external quality control in many laboratories in Poland and worldwide, considerable discrepancies between the obtained results are to be expected [5, 6], which may lower the effectiveness of the therapy.

In response to the abovementioned difficulties in the manual assessment of sperm, the first computer system for an automatized sperm analysis was designed over 30 years ago [7]. Repeatability of the measurements and their objectivism might constitute a potential advantage of the automatic systems, chief among them computer-assisted sperm analysis (CASA), which seems to be the most popular and most intensively improved tool over the years. CASA is a system combining specific hardware and software forms, a high-resolution camera, and a microscope. It is able to recognize the intensity and density of the pixels in the images taken on the appropriate background. After computer-assisted processing of the slides, it estimates sperm concentration, velocity and morphology. However, the sample preparation methods and settings recommended by the producer have to be strictly followed in order to obtain credible results.

In 1998, the Special Interest Group for Andrology of the European Society for Human Reproduction (ESHRE) released guidelines on the application of CASA in the analysis of spermatozoa [8]. The experts reported a borderline usefulness of CASA for the assessment of concentration and motility of human sperm. They also stated that CASA should not be used for the analysis of sperm morphology. Thus, manual examination of the sperm and the $\mathrm{WHO}$ guidelines should serve as a reference and the gold standard for sperm evaluation. In light of the fact that 20 years have passed since the ESHRE guidelines were released and that the CASA system underwent a series of improvements, it seems the time has come to reconsider the promising capacities of computer-assisted sperm analysis and to address its usefulness in clinical practice on a par with the manual assessment.

\section{OBJECTIVES}

The aim of the study was to check the quality of computer-assisted sperm analysis in comparison to the reference manual method as well as standardization of computer semen assessment using CASA.

\section{MATERIAL AND METHODS}

The study was conducted between January and June 2015 at the Andrology Laboratory of the Division of In- fertility and Reproductive Endocrinology, Poznań University of Medical Sciences, Poland. The study group included 230 men who gave sperm samples for the first time in our center as part of an infertility investigation. Azoospermia was an excluding criterion. All participants signed an informed written consent. Local Ethics Committee approved of the study (no. 766/13 and 855/13).

The participants provided sperm samples by masturbation after 2-5 days of sexual abstinence. Semen samples were collected into a standard container and examined after 30-60 min of liquefaction. First, the volume and $\mathrm{pH}$ of the samples were analyzed. Then, the samples underwent manual and computer-assisted analysis of concentration, motility and morphology.

A total of 184 samples were examined once manually and once using CASA. Forty-six samples underwent two manual analyses and two computer-assisted analyses, and served as the internal control. The measurements were performed in parallel, by two highly trained technicians, who reported their results on two separate forms.

\section{Manual sperm analysis}

The 2010 WHO Manual was strictly followed as a reference for the manual sperm examination [2]. Concentration was assessed using the Neubauer hemocytometer. Every analysis was performed twice. We referred the difference between obtained values to the deviation range allowed by $\mathrm{WHO}$.

Sperm motility was analyzed after liquefaction of the samples, twice for each sample. In accordance with the 2010 WHO guidelines, we described the following patterns of motility: progressive motility, non-progressive motility, and no motility, based on sperm velocity. In every examination, we assessed 5 fields of vision and counted 200 cells at $400 x$ magnification. We counted the mean of two obtained motility values and calculated the result with a $1 \%$ accuracy.

Sperm morphology was also analyzed twice for each sample. The smears were stained using the Papanicolaou method and examined under a microscope at 1000x magnification. Mean of the sperms with proper morphology from two analyses gave the final result.

\section{Computer-assisted sperm analysis}

CASA SCA (5.4, Microptic SL, Barcelona, Spain) is a computer system which requires a high-resolution camera connected to a phase-contrast microscope. The analysis of a single view-field takes 1 second. Sperm concentration, motility and morphology were examined with the provided CASA system. Each analyzed slide may be saved in system memory and the examination may be repeated.

Semen analysis was conducted with strict adherence to the program settings provided by the manufacturer [9]. 
CASA was borrowed from the producer for the time of the study. The settings of the program were installed and controlled by an Microptic representative, according to the Microptic CASA manual [9].

The samples were prepared and the examination was conducted according to the instruction. Leja 10/20 um CE chambers (Leja Products, Nieuw-Vennep, Netherlands) were used for the imaging. For each analysis, 200 spermatozoa were used. Manual and CASA motility measurements were performed simultaneously by two technicians to reduce the influence of time on sperm kinematics.

\section{Quality control}

Before the study, the technicians were trained to use the system by an employee of the Microptic company.

The Andrology Laboratory staff are certified in measuring sperm parameters and participate in the External Quality Assessment Program (EQAP) supervised by ESHRE. Since 2006, every half year the technicians examining semen samples undergo internal and external quality control.

\section{Statistical analysis}

The assumption concerning normality of the distribution of values of the analyzed sperm characteristics (checked using the Kolmogorov-Smirnov test) was not rejected. In consequence, the t-Student test for dependent variables was used to compare the results obtained manually and using CASA. The same test was used to compare the double measures within each of the assessment methods (internal control). The calculations were performed using Statistica 10 , StatSoft, Tulsa. The $p$-value of $p<0.05$ was considered as statistically significant.

\section{RESULTS}

Mean patient age was 34 (23-46, SD 5) years. Mean sperm parameters obtained with the manual (M) and computer-assisted (C) sperm analysis, as well as the comparison of the manually and CASA-obtained values are presented in Table 1. Statistically significant differences were found between both methods in all of the assessed sperm pa- rameters, except for non-progressive motility. Also standard deviation was higher for the computer-assisted analysis in all cases except for the assessment of non-progressive motility.

Furthermore, we compared the results of sperm parameters in the group where all analyses with each method were performed twice on the same sample (in each case $p>0.05$; Figure $1 \mathrm{~A}-\mathrm{F})$. No significant differences were found between both assessments of the same sample, neither manually nor with CASA. However, standard deviation was higher in the CASA group.

The graphs for differences of the analyzed sperm parameters for repeated measurements (for 46 men) show their proximity to 0 (no difference). The statistical testing was performed for manual and computer measurements independently.

The differences were obtained for all parameters by subtracting the second measurement from the first measurement (which obviously could be done in the opposite direction).

There are no statistically significant differences for all of the assessed sperm parameters in each of the two (manual and computer) measurements (for all cases $p>0.05$ ).

\section{DISCUSSION}

The importance of seminological analysis in the diagnostic process and treatment of male infertility remains indisputable. According to the WHO and the ESHRE manual, semen examination is a gold standard for defining the role of men in infertility $[2,8]$. Sperm parameters obtained in accordance with the $\mathrm{WHO}$ recommendations remain a reference for the clinical practice. However, they do not always express the fertilizing potential of a male. Our results along with those presented in the previous publications, show that computer-assisted sperm analysis in human reproduction centers requires further studies.

In order to maximize the objectivity of our study, we introduced an internal quality control of the results. We performed two independent assessments of the samples using both, computer-assisted and manual methods in 46 cases, and compared the results within each method. No significant differences were observed between the main samples and controls, neither for CASA, nor for the manual analy-

Table 1. Comparison of sperm parameters obtained by manual and CASA analysis

\begin{tabular}{|c|c|c|c|c|c|c|c|c|c|c|}
\hline & \multicolumn{2}{|c|}{$\begin{array}{l}\text { Concentration } \\
{[\mathrm{m} / \mathrm{n} / \mathrm{mL}]}\end{array}$} & \multicolumn{2}{|c|}{$\begin{array}{l}\text { Progressive motility } \\
{[\%]}\end{array}$} & \multicolumn{2}{|c|}{$\begin{array}{c}\text { Non-progressive } \\
\text { motility [\%] }\end{array}$} & \multicolumn{2}{|c|}{ No motility [\%] } & \multicolumn{2}{|c|}{ Morphology [\%] } \\
\hline & M & C & M & C & M & C & M & C & M & C \\
\hline Mean & 34 & 39 & 30 & 34 & 29 & 29 & 40 & 36 & 3 & 4 \\
\hline SD & 29 & 32 & 16 & 19 & 12 & 9 & 20 & 21 & 1 & 4 \\
\hline$p$ & \multicolumn{2}{|c|}{$<0.0001$} & \multicolumn{2}{|c|}{$<0.0001$} & \multicolumn{2}{|c|}{$>0.05$} & \multicolumn{2}{|c|}{$<0.05$} & \multicolumn{2}{|c|}{$<0.0001$} \\
\hline $\mathrm{n}$ & \multicolumn{10}{|c|}{230} \\
\hline
\end{tabular}

CASA — computer-assisted sperm analysis; M — manual assessment according to WHO, 2010, C — computer assessment with CASA; SD — standard deviation 
A

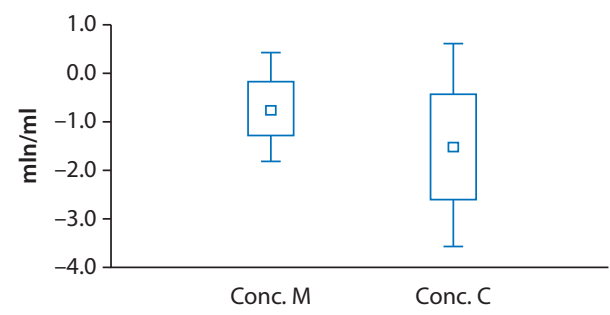

C

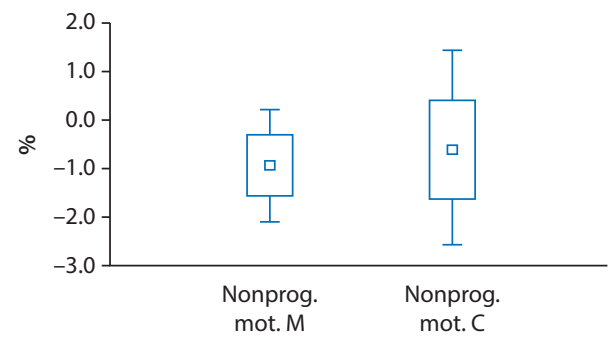

E

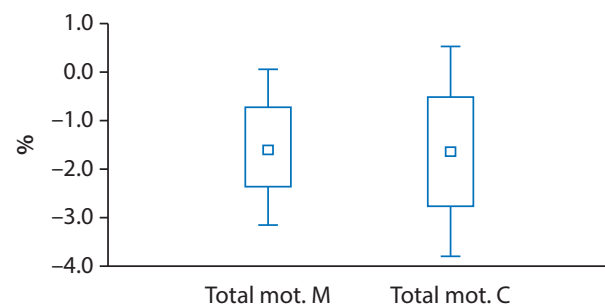

B

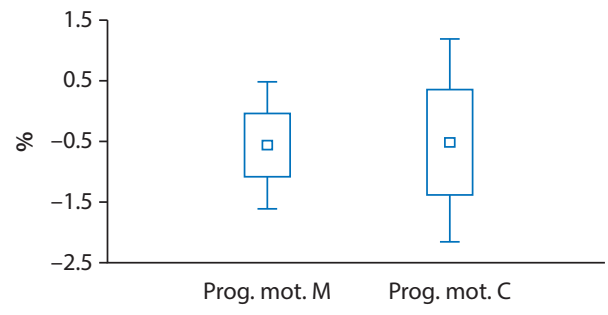

D

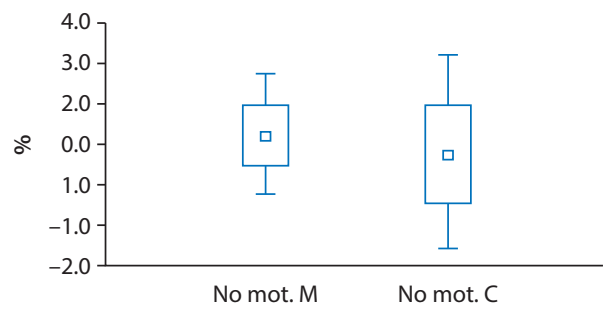

F

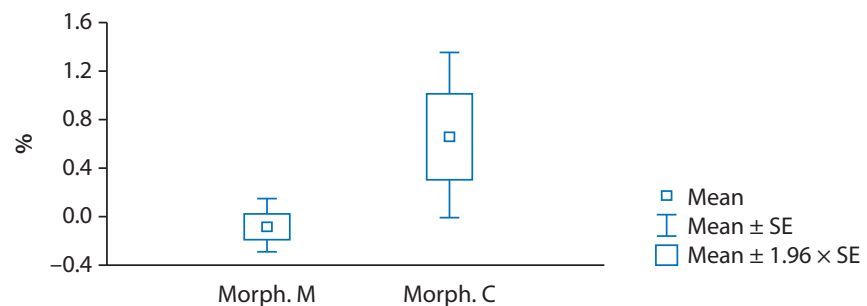

Figure 1. Box and Whisker plot of differences of $\mathbf{A}$ - sperm concentration for repeated manual (M) and computer (C) measurements; $\mathbf{B}$ - sperm progressive motility for repeated manual $(M)$ and computer $(C)$ measurements; $\mathbf{C}$ - sperm non-progressive motility for repeated manual (M) and computer (C) measurements; D - sperm no motility for repeated manual (M) and computer (C) measurements; $\mathbf{E}$ - total sperm motility for repeated manual $(\mathrm{M})$ and computer $(\mathrm{C})$ measurements; $\mathbf{F}$ - sperm morphology for repeated manual (M) and computer (C) measurements

sis. However, we observed higher standard deviation for the samples assessed using the computer method. Therefore, we hypothesized that the computer-assisted analysis might be biased by an individual interpretation of the technician. The parallel assessment of the samples by two technicians might be the limitation of the study. On the other hand, by regular and successful participation in EQAP, this bias might be considered as not significant. Furthermore, the staff have been thoroughly trained by the manufacturers representative, so their level of competence was very good. We cannot rule out that variability within the CASA system could also influence the obtained results [10]. The technicians who use CASA do not have to specialize in semen analysis but they need to undergo specialist training [11]. However, they should have experience in seminology to perform the analysis properly.

Strict adherence to the producers settings of CASA and the use of Leja chambers for human sperm assessment are extremely important to obtain reliable results $[3,4]$. In our study, we fulfilled these conditions but we still observed discrepancies between the computer results and manual reference. Concentrations measured with CASA were significantly higher as compared to the manual control. In contrast, Lammers et al., reported that the overall concentration did not significantly differ between automatic assessment and manual control [11]. However, as in case of our findings, CASA values were higher than these obtained manually, as were the standard deviations. Bearing in mind the clinical importance of adequate examination of the number of sperms for therapeutic decisions in infertile couples [12], these observations further support the need for caution when using CASA in human reproduction medicine.

Almost all of the motility results obtained using CASA, except for non-progressive motility, were significantly different as compared to the manual analysis. All CASA values 
were higher than the parameters obtained manually, which is consistent with the study of Cooper et al. [13]. These authors compared the motility obtained manually and using the computer-assisted method over a period of 43 months. They found that with time the values assessed with both methods became similar, which might prove the importance of training and experience with the automatic system when the final result is concerned. In our study, in order to compare the samples at the similar kinetics, manual and CASA analysis were performed in parallel. Regardless experience and training of the technicians, the observed progressive motility values were significantly higher in case of CASA than the manual examination. The results of both studies might reveal the next limitation of CASA, namely that sperm motility might be overestimated because the system is not able to perfectly differentiate the velocity of the gametes when they cross. The system is not able to assess flagellar beating properly [4]. That is why non-progressive motility values obtained in computer-assisted analysis are comparable to the manual values, while at the same time non-motile cells are estimated as higher due to the cellular debris present on the slides.

Morphology, the most subjective marker, was also assessed in our study as higher using CASA, with significantly higher standard deviation values than in the manual analysis. Furthermore, automatic morphology assessment was more time consuming than the manual analysis. Lammers et al., reported a significantly lower computer-obtained morphology than in manual controls.

In Poland, there are only a few laboratories which participate in the external and internal quality control programs. Presumably, the proportion worldwide is similar. Laboratories without quality controls may make numerous mistakes in the complicated process of human sperm analysis $[3,5,14]$. Furthermore, the results of sperm analysis do not precisely correlate with fertility of a patient, but more show the probability of male factor of infertility [12]. Therefore it might be debatable, to which extent the differences observed in results obtained using both methods are relevant for the clinical practice. CASA might offer a chance to improve the repeatability and objectivity of sperm parameters. On the other hand, the abovementioned limitations of CASA had been known before and various producers have been focusing on improving the system by including better Brownian motion filtering, illumination control, introducing fractals as a kinematic measure, and others [4].

\section{CONCLUSIONS}

The CASA system offers considerable advantages over the manual assessment, i.e. speed (especially in sperm motility and concentration measurements), automatic data capture, and relatively short training for the technicians. On the other hand, the discrepancies between the results of the automatic versus manual assessment, as well as considerable amount of time needed for sperm morphology assessments still require further developments for the use of CASA in clinical practice. However, it might be used for screening examinations in the laboratories without internal and external quality control programs.

\section{ACKNOWLEDGEMENTS}

The authors wish to express their sincere gratitude to professor Gerhard van der Horst for his invaluable suggestions and Microptic SL, Barcelona, Spain, for delivering SCA-5.4 system for the analysis.

\section{REFERENCES}

1. Kuczyński W, Kurzawa R, Oszukowski P, et al. Polish Gynecological Society and Polish Society for Reproductive Medicine. [Polish Gynecological Society and Polish Society for Reproductive Medicine recommendations for the diagnosis and treatment of infertility]. Ginekol Pol. 2012; 83(2): 149-154, indexed in Pubmed: 22568363.

2. WHO. World Health Organization reference values for human semen characteristics. Cambridge University Press, Cambridge. ; 2010.

3. Bailey E, Fenning N, Chamberlain S, et al. Validation of sperm counting methods using limits of agreement. J Androl. 2007; 28(3): 364-373, doi: 10.2164/jandrol.106.002188, indexed in Pubmed: 17215545.

4. Mortimer ST, van der Horst G, Mortimer D. The future of computer-aided sperm analysis. Asian J Androl. 2015; 17(4): 545-553, doi: 10.4103/1008682X.154312, indexed in Pubmed: 25926614.

5. Walczak-Jedrzejowska R, Marchlewska K, Oszukowska E, et al. Semen analysis standardization: is there any problem in Polish laboratories? Asian J Androl. 2013; 15(5): 616-621, doi: 10.1038/aja.2013.48, indexed in Pubmed: 23817502

6. Jedrzejczak P, Talarczyk J, Taszarek-Hauke G, et al. [External quality assessment of semen analysis in Poland]. Ginekol Pol. 2012; 83(11): 835-840, indexed in Pubmed: 23379191.

7. Kaskar K, Franken DR, van der Horst G, et al. The effect of pentoxifylline on sperm movement characteristics and zona pellucida binding potential of teratozoospermic men. Hum Reprod. 1994; 9(3): 477-481, indexed in Pubmed: 8006137.

8. Guidelines on the application of CASA technology in the analysis of spermatozoa. ESHRE Andrology Special Interest Group. European Society for Human Reproduction and Embryology. Hum Reprod. 1998; 13(1): 142-145, indexed in Pubmed: 9512246.

9. Systems M-AD. SCA - Sperm Class Analyzer v. 4.1. - polish version: Microptic - Automatic Diagnostic Systems. ; 2014.

10. Holt W, Watson P, Curry M, et al. Reproducibility of computer-aided semen analysis: comparison of five different systems used in a practical workshop. Fertil Steril. 1994; 62(6): 1277-1282, indexed in Pubmed: 7958000.

11. Lammers J, Splingart $C$, Barrière $P$, et al. Double-blind prospective study comparing two automated sperm analyzers versus manual semen assessment. J Assist Reprod Genet. 2014; 31(1): 35-43, doi: 10.1007/s10815013-0139-2, indexed in Pubmed: 24242989.

12. Jedrzejczak P, Taszarek-Hauke G, Hauke J, et al. Prediction of spontaneous conception based on semen parameters. Int J Androl. 2008; 31(5): 499-507, doi: 10.1111/j.1365-2605.2007.00799.x, indexed in Pubmed: 17651398.

13. Cooper TG, Yeung $\mathrm{CH}$. Computer-aided evaluation of assessment of "grade a" spermatozoa by experienced technicians. Fertil Steril. 2006; 85(1): 220-224, doi: 10.1016/j.fertnstert.2005.07.1286, indexed in Pubmed: 16412757.

14. Keel BA, Quinn P, Schmidt CF, et al. Results of the American Association of Bioanalysts national proficiency testing programme in andrology. Hum Reprod. 2000; 15(3): 680-686, indexed in Pubmed: 10686219. 